\title{
ON FIXED POINTS OF DOUBLY SYMMETRIC RIEMANN SURFACES
}

\author{
GRZEGORZ GROMADZKI* \\ Institute of Mathematics, University of Gdańsk, Wita Stwosza 57, Gdańsk 80-952, Poland \\ e-mail: greggrom@math.univ.gda.pl \\ and EWA KOZŁOWSKA-WALANIA** \\ Institute of Mathematics, University of Gdańsk, Wita Stwosza 57, Gdańsk 80-952, Poland \\ e-mail: retrakt@math.univ.gda.pl
}

(Received 23 June 2006; revised 25 January 2008; accepted 1 February 2008)

\begin{abstract}
In this paper, we study ovals of symmetries and the fixed points of their products on Riemann surfaces of genus $g \geq 2$. We show how the number of these points affects the total number of ovals of symmetries. We give a generalisation of Bujalance, Costa and Singerman's theorems in which we show upper bounds for the total number of ovals of two symmetries in terms of $g$, the order $n$ and the number $m$ of the fixed points of their product, and we show their attainments for $n$ holding some divisibility conditions. Finally, we give an upper bound for $m$ in terms of $n$ and $g$, and we study conditions under which it has given parity.
\end{abstract}

2000 Mathematics Subject Classification. Primary 30F; Secondary 14H.

1. Introduction. Let $X$ be a compact Riemann surface of genus $g>1$. By a symmetry of $X$ we mean, in this paper, an antiholomorphic involution $a$ of $X$, which has fixed points. By the classical result of Harnack, the set of fixed points of $a$ consists of at most $g+1$ disjoint simple closed curves, which, following classical Hilbert terminology, are called ovals. If $a$ has $g+1-q$ ovals, then following Natanzon [5], we shall call it an $(M-q)$-symmetry.

In [1] (see also [2]), the bounds for the total number of ovals of two symmetries in terms of $g$ and the order $n$ of their product were given. Here, using a theorem of Macbeath from [4] and a result from [6], we give a generalisation of these results, which takes into account the number $m$ of the fixed points of the product of symmetries. We also show the sharpness of our bounds for infinitely many $n$.

In the remainder of the work, we focus attention on possible values of $m$ for given $n$ and $g$. We find an upper bound for it and we study its attainments. Finally, we look for the conditions that guarantee specified parity of $m$.

2. Preliminaries. We shall prove our results using the theory of non-Euclidean crystallographic groups (NEC groups in short) by which we mean discrete and cocompact subgroups of the group $\mathcal{G}$ of all isometries of the hyperbolic plane $\mathcal{H}$.

\footnotetext{
* Supported by the Grant SAB 2005-0049 of the Spanish Ministry of Eduction and Sciences.

**Supported by BW 5100-5-0198-6.
} 
The algebraic structure of such group $\Lambda$ is determined by the signature:

$$
s(\Lambda)=\left(g ; \pm ;\left[m_{1}, \ldots, m_{r}\right] ;\left\{\left(n_{11}, \ldots, n_{1 s_{1}}\right), \ldots,\left(n_{k 1}, \ldots, n_{k s_{k}}\right)\right\}\right),
$$

where the brackets $\left(n_{i 1}, \ldots, n_{i s_{i}}\right)$ are called the period cycles, the integers $n_{i j}$ are the link periods, $m_{i}$ proper periods, and finally, $g$ the orbit genus of $\Lambda$.

A group $\Lambda$ with signature (1) has the presentation with the following generators, called canonical generators:

$x_{1}, \ldots, x_{r}, e_{i}, c_{i j}, 1 \leq i \leq k, 0 \leq j \leq s_{i}$ and $a_{1}, b_{1}, \ldots, a_{g}, b_{g}$ if the sign is + or $d_{1}, \ldots, d_{g}$ otherwise,

and relators:

$x_{i}^{m_{i}}, i=1, \ldots, r, c_{i j}^{2},\left(c_{i j-1} c_{i j}\right)^{n_{i j}}, c_{i 0} e_{i}^{-1} c_{i s_{i}} e_{i}, i=1, \ldots, k, j=0, \ldots, s_{i}$

and

$$
x_{1} \cdots x_{r} e_{1} \cdots e_{k} a_{1} b_{1} a_{1}^{-1} b_{1}^{-1} \cdots a_{g} b_{g} a_{g}^{-1} b_{g}^{-1} \text { or } x_{1} \cdots x_{r} e_{1} \cdots e_{k} d_{1}^{2} \cdots d_{g}^{2},
$$

according to whether the sign is + or - . The elements $x_{i}$ are elliptic transformations $a_{i}, b_{i}$ hyperbolic translations, $d_{i}$ glide reflections and $c_{i j}$ hyperbolic reflections. Every element of finite order in $\Lambda$ is conjugate either to a canonical reflection or to a power of some canonical elliptic element $x_{i}$ or else to a power of the product of two consecutive canonical reflections.

Now an abstract group with such presentation can be realized as an NEC group $\Lambda$ if and only if the value

$$
2 \pi\left(\varepsilon g+k-2+\sum_{i=1}^{r}\left(1-\frac{1}{m_{i}}\right)+\frac{1}{2} \sum_{i=1}^{k} \sum_{j=1}^{s_{i}}\left(1-\frac{1}{n_{i j}}\right)\right),
$$

where $\varepsilon=2$ or 1 according to the sign being + or - is positive. This value turns out to be the hyperbolic area $\mu(\Lambda)$ of an arbitrary fundamental region for such group, and we have the following Hurwitz-Riemann formula:

$$
\left[\Lambda: \Lambda^{\prime}\right]=\mu\left(\Lambda^{\prime}\right) / \mu(\Lambda)
$$

for a subgroup $\Lambda^{\prime}$ of finite index in an NEC group $\Lambda$.

Now NEC groups having no orientation reversing elements are classical Fuchsian groups. They have signatures $\left(g ;+;\left[m_{1}, \ldots, m_{r}\right] ;\{-\}\right)$, which shall be abbreviated as $\left(g ; m_{1}, \ldots, m_{r}\right)$. Given an NEC group $\Lambda$, the subgroup $\Lambda^{+}$of $\Lambda$ consisting of the orientation-preserving elements is called the canonical Fuchsian subgroup of $\Lambda$ and for a group with signature (1), it has, by [7], signature

$$
\left(\varepsilon g+k-1 ; m_{1}, m_{1}, \ldots, m_{r}, m_{r}, n_{11}, \ldots, n_{k s_{k}}\right) .
$$

A torsion-free Fuchsian group $\Gamma$ is called a surface group and it has signature $(g ;-)$. In such case, $\mathcal{H} / \Gamma$ is a compact Riemann surface of genus $g$ and conversely, each compact Riemann surface can be represented as such orbit space for some $\Gamma$. Furthermore, given a Riemann surface so represented, a finite group $G$ is a group of automorphisms of $X$ if and only if $G=\Lambda / \Gamma$ for some NEC group $\Lambda$. 
Let $C(G, g)$ denote the centralizer of an element $g$ in $G$. The following result from [6] and the next theorem of Macbeath from [4] are crucial for the paper.

THEOREM 2.1. Let $X=\mathcal{H} / \Gamma$ be a Riemann surface with a group $G$ of all automorphisms of $X$, let $G=\Lambda / \Gamma$ for some NEC group $\Lambda$ and let $\theta: \Lambda \rightarrow G$ be the canonical epimorphism. Then, the number of ovals of a symmetry a of $X$ equals

$$
\sum\left[C\left(G, \theta\left(c_{i}\right)\right): \theta\left(C\left(\Lambda, c_{i}\right)\right)\right]
$$

where the sum is taken over a set of representatives of all conjugacy classes of canonical reflections whose images under $\theta$ are conjugate to a.

For a symmetry $a$, we shall denote by $\|a\|$ the number of its ovals. The index $w_{i}=\left[C\left(G, \theta\left(c_{i}\right)\right): \theta\left(C\left(\Lambda, c_{i}\right)\right)\right]$ will be called a contribution of $c_{i}$ to $\|a\|$.

COROLlARY 2.2. Let $\mathrm{D}_{n}=\Lambda / \Gamma$ be the group of automorphisms of a Riemann surface $X=\mathcal{H} / \Gamma$ generated by two non-central symmetries $a$ and $b$ and let $C=\left(n_{1}, \ldots, n_{s}\right)$ be a period cycle of $\Lambda$. If $n$ is odd, then the reflections corresponding to $C$ contribute to $\|a\|$ and $\|b\|$ with at most 2 ovals in total. If $n$ is even, then the reflections corresponding to $C$ contribute to $\|a\|$ and $\|b\|$ with at most tovals in total, where $t$ is the number of even link periods if $s \geq 1$ and some $n_{i}$ is even and with at most 2 ovals in total for the remaining cases.

Proof. Let $\theta: \Lambda \rightarrow \mathrm{D}_{n}$ be the canonical epimorphism. The case of odd $n$ is trivial; here all canonical reflections belonging to $C$ are conjugate, $C\left(\mathrm{D}_{n}, \theta(c)\right)$ has order 2 and $c \in C(\Lambda, c)$.

Now for $n$ even, the centralizer of any non-central element of $\mathrm{D}_{n}$ has order 4 . Since $c_{i} \in C\left(\Lambda, c_{i}\right)$, we have that $w_{i} \leq 2$ and since $a$ and $b$ are not conjugate, we can assume that $s \geq 2$ or $s=1$ and $n_{1}$ is even. If $c$ belongs to two odd link periods, then we can assume that $c$ neither contributes to $\|a\|$ nor to $\|b\|$, while if $c$ belongs to an even link period $n_{1}$ and $c c^{\prime}$ has order $n_{1}$, then $\left(c c^{\prime}\right)^{n_{1} / 2} \in C(\Lambda, c)$. Now $\theta\left(\left(c c^{\prime}\right)^{n_{1} / 2} c\right) \neq 1$ since $\operatorname{ker} \theta$ is a Fuchsian group, and therefore, we see that $\theta(C(\Lambda, c))$ has order 4 .

We also need the following result of Macbeath from [4] concerning the number of fixed points of an automorphism of a Riemann surface (c.f. [3] for the case of nonorientable Riemann surfaces). By $N_{G}(\langle g\rangle)$, we mean the normalizer in $G$ of the group generated by $g$.

THEOREM 2.3. Let $G=\Delta / \Gamma$ be the group of orientation preserving automorphisms of a Riemann surface $X=\mathcal{H} / \Gamma$ and let $x_{1}, x_{2}, \ldots, x_{r}$ be the set of canonical elliptic generators of $\Delta$ with periods $m_{1}, \ldots, m_{r}$, respectively. Let $\theta: \Lambda \rightarrow G$ be the canonical epimorphism. Then, the number $m$ of points of $X$ fixed by $g \in G$ is given by the formula

$$
m=\left|N_{G}(\langle g\rangle)\right| \sum 1 / m_{i},
$$

where the sum is taken over those $i$ for which $g$ is conjugate to a power of $\theta\left(x_{i}\right)$.

We shall study the number of fixed points of the product of two symmetries $a$ and $b$ of a Riemann surface $X$, which has the order $n$. Let $X=\mathcal{H} / \Gamma$ and $\langle a, b\rangle=\Lambda / \Gamma$ for some NEC group $\Lambda$ with signature (1) and Fuchsian surface group $\Gamma$. Let $r$ and $s$ denote, respectively, the numbers of proper periods and link periods equal to $n$ in the signature of $\Lambda$. The subgroup of $\Lambda / \Gamma$ of orientation preserving automorphisms is 
generated by the product $a b$ and is $\Lambda^{+} / \Gamma$. Hence, using the above theorem and (2), we obtain the following.

COROLlary 2.4. The product of two symmetries $a$ and $b$ of a Riemann surface $X$, whose order is equal to $n$, has $2 r+s$ fixed points.

3. On ovals of two symmetries with specified number of fixed points of their product. Here we study how the number $m$ of the fixed points of the product of two symmetries of a Riemann surface of genus $g$ affects the total number $t$ of their ovals, and we give upper bounds for $t$ depending on the parity of $n$. We also show that, with some small exceptions, our bounds are sharp for arbitrary arithmetically admissible $m, n$ and $g \geq 2$, that is for $n$ dividing $2 g+m-2$. Throughout the remainder of the paper, $a$ and $b$ will denote two symmetries whose product has order $n$ and has $m$ fixed points.

THEOREM 3.1. Two symmetries $a$ and $b$ of Riemann surface $X$ of genus $g$ whose product has order $n$ and has $m$ fixed points have at most

$$
4 g / n+m-2(m-2)(n-1) / n
$$

ovals in total.

Proof. Let $t$ denote total number of ovals of $a$ and $b$ and let $G=\langle a, b\rangle=\mathrm{D}_{n}$. Now $G=\Lambda / \Gamma$ for some surface Fuchsian group $\Gamma$ and an NEC group $\Lambda$ with signature

$$
\left(h ; \pm ;\left[m_{1}, \ldots, m_{r}\right] ;\left\{C_{1}, \ldots, C_{k},\left(n_{1}\right), \ldots,\left(n_{l}\right),(-), . . . .,(-)\right\}\right),
$$

where $C_{i}=\left(n_{i 1}, \ldots, n_{i s_{i}}\right)$ with $s_{i} \geq 2$ or $s_{i}=1$ and $n_{i 1}$ even and $n_{1}, \ldots, n_{l}$ are odd. Throughout the paper, let $p=\varepsilon h+k+l+u-1$, where $\varepsilon=2$ or 1 according to the sign of $\Lambda$ being + or - ; it is known as the algebraic genus of $\Lambda$. Let $s=s^{\prime}+s^{\prime \prime}$ where $s^{\prime}$ denotes number of link periods $n$ and similarly let $r=r^{\prime}+r^{\prime \prime}$ where $r^{\prime}$ denotes number of proper periods equal $n$. Now by Corollary 2.4 , we have $m=2 r^{\prime}+s^{\prime}$. Also $t \leq 2 u+2 l+s^{\prime}+s^{\prime \prime}$ by Corollary 2.2 , and thus,

$$
\begin{aligned}
2 \pi(g-1) / n & =\mu(\Lambda) \\
& \geq 2 \pi\left(p-1+r^{\prime}(1-1 / n)+r^{\prime \prime} / 2+s^{\prime}(1-1 / n) / 2+s^{\prime \prime} / 4\right) \\
& \geq 2 \pi\left(-1+(1-1 / n) m / 2+r^{\prime \prime} / 2+s^{\prime \prime} / 4+(u+l) / 2\right) \\
& \geq 2 \pi\left(-1+(1-1 / n) m / 2+r^{\prime \prime} / 2+\left(t-s^{\prime}\right) / 4\right) \\
& \geq 2 \pi\left(-1+(1-1 / n) m / 2+r^{\prime \prime} / 2+t / 4-m / 4\right) \\
& \geq(\pi / 2)(-4+2 m(1-1 / n)+t-m)
\end{aligned}
$$

which gives $t \leq 4(g-1) / n+4+m-2 m+2 m / n=(4 g+2 m-4) / n+4-m=4 g /$ $n+m-2(m-2)(n-1) / n$.

THEOREM 3.2. The bound in the previous theorem is attained for every $g, m \geq 2$ and every even $n$ such that $2 g+m \equiv 2 \bmod n$.

Proof. Let $\Lambda$ be an NEC group with signature

$$
(0 ;+;[-] ;\{(2, . . s ., 2, n, . \stackrel{m}{.}, n)\}),
$$


where $s=(4 g+2 m-4) / n+4-2 m$ and consider an epimorphism $\theta: \Lambda \rightarrow \mathrm{D}_{n}=$ $\left\langle a, b \mid a^{2}, b^{2},(a b)^{n}\right\rangle$ defined by $\theta\left(e_{1}\right)=1$ and which sends consecutive canonical reflections corresponding to the period cycle into

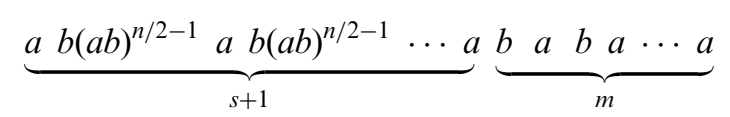

So by the Hurwitz-Riemann formula for $\Gamma=\operatorname{ker} \theta, X=\mathcal{H} / \Gamma$ has genus $g$ and by Theorem 2.1, symmetries $a$ and $b$ have $4 g / n+m-2(m-2)(n-1) / n$ ovals in common.

COROLlary 3.3. The bound from Theorem 3.1 is not attained for $m=0$ and $m=1$.

Proof. By [1], the total number of ovals of two such symmetries does not exceed $4 g / n+2$. On the other hand, for $m \leq 1,4 g / n+m-2(m-2)(n-1) /$ $n>4 g / n+2$.

If $m=1, n=2$, then the total number of ovals is $<2 g+(3 / 2)$ and the next theorem deals with the case $m=0, n=2$.

THEOREM 3.4. Two commuting symmetries, whose product does not have fixed points have at most $g+3$ ovals in total and this bound is attained for every odd $g>2$. The product of commuting symmetries on a Riemann surface of even genus has fixed points.

Proof. We know that $G=\mathrm{D}_{2}=\Lambda / \Gamma$ and as $m=0$ a group $\Lambda$ has signature

$$
(h ; \pm ;[-] ;\{(-), . l .,(-)\}),
$$

by Corollary 2.4, where $\varepsilon h+l \geq 3$ as $\mu(\Lambda)>0$. By the Corollary 2.2, we have $t \leq$ $2 l$ and also we know that $\pi(g-1)=\mu(\Gamma) / 4=\mu(\Lambda)=2 \pi(\varepsilon h+l-2) \geq \pi(2 l-4) \geq$ $\pi(t-4)$. So the first statement follows and also we see that $g$ is odd as $g=2 \varepsilon h+2 l-3$.

To show the attainment of this bound for odd $g>2$, consider an NEC group $\Lambda$ with signature $(0 ;+;[-] ;\{(-), . . .,(-)\})$ where $l=(g+3) / 2$ and an epimorphism $\theta: \Lambda \rightarrow \mathrm{D}_{2}$ that sends all $e_{i}$ into 1 and canonical reflections alternatively to $a$ and $b$. As each period cycle produces two ovals in $a$ or $b$, by Theorem 2.1, $\theta$ defines desired configuration of symmetries.

Now we will show that, like in [1], the bound in Theorem 3.1 can be significantly improved for odd $n$.

THEOREM 3.5. Two symmetries $a$ and $b$ of a Riemann surface of genus $g$, whose product has odd order $n$ and has $m$ fixed points have at most

$$
2(g-1) / n+4-m(n-1) / n
$$

ovals in total.

Proof. As in the proof of Theorem 3.1, we have $G=\langle a, b\rangle=\mathrm{D}_{n}=\Lambda / \Gamma$ for some surface Fuchsian group $\Gamma$ and an NEC group $\Lambda$ with signature (3), where $s_{i} \geq 2$. Now by Corollary 2.2, we have $t \leq 2 k+2 l+2 u$ and so

$$
\begin{aligned}
2 \pi(g-1) / n & =\mu(\Lambda) \\
& \geq 2 \pi\left(\varepsilon h+l+k+u-2+r^{\prime}(1-1 / n)+s^{\prime}(1-1 / n) / 2\right) \\
& \geq 2 \pi(\varepsilon h+t / 2-2+(1-1 / n) m / 2) \\
& \geq \pi(-4+t+m(1-1 / n))
\end{aligned}
$$

which gives $t \leq 2(g-1) / n+m / n+4-m=2(g-1) / n+4-m(n-1) / n$. 
THEOREM 3.6. The bound in the previous theorem is attained for every $m, n$ and $g \geq 2$ for which $2 g+m \equiv 2 \bmod n$.

Proof. Let $\Lambda$ be an NEC group with signature

$$
(0 ;+;[-] ;\{(n, . m ., n),(-), \stackrel{l-1}{.},(-)\})
$$

where $l=(g+m / 2-1) / n+2-m / 2$. Consider an epimorphism $\theta: \Lambda \rightarrow G$ defined by $\theta\left(c_{i 0}\right)=a$ for all $i>1, \theta\left(e_{i}\right)=1$ for all $i$ and sending canonical reflections corresponding to the non-empty period cycle alternatively to $a$ and $b$ starting with $a$ for even $m$ and if $m$ is odd defined on all canonical generators in the same way as before except $\theta\left(c_{1 m-1}\right)=a b a$ with $\theta\left(c_{1 m}\right)=a$. This gives rise to the desired configuration of symmetries.

COROLLARY 3.7. If a and $b$ are two non-commuting symmetries of a Riemann surface of genus $g$ whose product has $m$ fixed points, then the total number of their ovals does not exceed $g+3-m / 2$.

Proof. It follows directly from Theorems 3.1 and 3.5.

By the degree of hyperellipticity of a conformal involution $\rho$ of a Riemann surface $X$, we shall understand the genus of the orbit space $X / \rho$.

COROllary 3.8. Two $(M-q)$ - and $\left(M-q^{\prime}\right)$-symmetries of a Riemann surface of genus $g$, whose product has $m$ fixed points, commute for $g \geq q+q^{\prime}+2-m / 2$. Furthermore, in such case, $m=2 g+2-4 p$, where $p$ denotes the degree of hyperellipticity of the involution $a b$.

Proof. Assume to a contrary that these symmetries do not commute. Then, we have $2 g+2-q-q^{\prime} \leq g+3-m / 2$ and so $g \leq q+q^{\prime}+1-m / 2$. Now if $a$ and $b$ are two commuting symmetries of a Riemann surface of genus $g$, then $G=\langle a, b\rangle=\mathrm{D}_{2}=\Lambda / \Gamma$ for some surface Fuchsian group $\Gamma$ and an NEC group $\Lambda$ with signature

$$
\left(h ; \pm ;[2, . r ., 2] ;\left\{\left(2, . s_{1} ., 2\right), \ldots,\left(2, s_{k} ., 2\right),(-) . l .,(-)\right\}\right) .
$$

Since the algebraic genus $p$ of $\Lambda$ is just the degree of hyperellipticity of $a b$, then for $p=\varepsilon h+k+l-1$, we have $\pi(g-1)=\mu(\Gamma) / 4=\mu(\Lambda)=2 \pi(p-1+m / 4)=\pi(2 p-$ $2+m / 2)$ and so $m=2 g+2-4 p$.

\section{On the number of fixed points of the product of two symmetries.}

THEOREM 4.1. Let $a$ and $b$ be two symmetries of a Riemann surface $X$ of genus $g$ whose product has order $n$. Then, ab has at most $2(g+n-1) /(n-1)$ fixed points.

Proof. As before, let $G=\langle a, b\rangle=\mathrm{D}_{n}=\Lambda / \Gamma$ for some surface Fuchsian group $\Gamma$ and an NEC group $\Lambda$ with signature (3). Then,

$$
\begin{aligned}
2 \pi(g-1) / n & =\mu(\Lambda) \\
& \geq 2 \pi\left(p-1+r^{\prime}(1-1 / n)+r^{\prime \prime} / 2+s^{\prime}(1-1 / n) / 2+s^{\prime \prime} / 4\right) \\
& \geq 2 \pi(-1+(1-1 / n) m / 2) \\
& =\pi(-2+m(1-1 / n) .
\end{aligned}
$$

So the statement follows since $m \leq(2(g-1) / n+2)(n /(n-1))=2(g+n-1) /$ $(n-1)$ 
From the next theorem, it follows in particular that the above bound is attained for arbitrary arithmetically admissible $g$ and $n$ with $n$ even.

THEOREM 4.2. Given $g \geq 2, m$, an even $n$ such that $g \equiv 0 \bmod (n-1)$ and $2(g+$ $n-1) /(n-1) \equiv m \bmod 4$, there exists a Riemann surface $X$ of genus $g$ having two symmetries whose product has order $n$ and has $m$ fixed points.

Proof. Let $2 g /(n-1)+2$ be denoted as $M$ and so $m=M-4 k$ for some integer $k$. Consider an NEC group $\Lambda$ with signature

$$
\left(0 ;+;[-] ;\left\{(-)^{k},(n, . \stackrel{m}{.}, n, n / 2, .2 k, n / 2)\right\}\right)
$$

and an epimorphism $\theta: \Lambda \rightarrow \mathrm{D}_{n}=\langle a, b\rangle$ defined by $\theta\left(e_{i}\right)=1$ for all $i, \theta\left(c_{i 0}\right)=a$ for reflections corresponding to empty period cycles and which sends canonical reflections corresponding to the non-empty period cycle onto

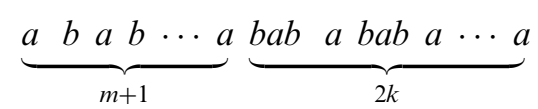

Here again by the Hurwitz-Riemann formula, we have get a Riemann surface of genus $g$ that admits two symmetries $a$ and $b$ whose product has $m$ fixed points.

Now we shall give some conditions under which $m$ have specified parity. Particularly interesting is the case when the product of symmetries has an odd number of fixed points.

THEOREM 4.3. If $n$ is a power of 2, then $m$ is even. If $n$ is even but is not a power of 2 , then for infinitely many $g$, there exists a Riemann surface of genus $g$ having two symmetries, whose product has order $n$ and $m$ is odd.

Proof. Let as always $G=\langle a, b\rangle=\Lambda / \Gamma$ and let $\theta: \Lambda \rightarrow G$ be the corresponding epimorphism. If there are no link periods equal to $n$, then by Corollary $2.4, m=2 r$ for $r$ being the number of proper periods equal to $n$ in the signature of $\Lambda$. So we can assume that there are link periods $n$. As both symmetries have ovals, the order of symmetry conjugate to $a$ and symmetry conjugate to $b$ is $n$ and $\theta\left(c_{i 0}\right)$ is conjugate to $\theta\left(c_{i s_{i}}\right)$, the number of link periods $n$ is even in each of the non-empty period cycles. Hence, the number of fixed points of $a b$ is even by 2.4.

For the second part, let $k \neq 1$ be the smallest odd divisor of $n$. Given an integer $u$ consider an NEC group $\Lambda$ with signature

$$
(0 ;+;[n, . . . ., n, \mu] ;\{(n, k, k, n / k)\}),
$$

where $\mu=n / \operatorname{gcd}(n, u+(k-1) / 2)$ and an epimorphism given by $\theta\left(x_{i}\right)=a b$ for $i=1, \ldots, u, \theta\left(x_{u+1}\right)=(b a)^{u+(k-1) / 2}, \theta(e)=(a b)^{(k-1) / 2}$ and which sends reflections corresponding to non-empty period cycle to

$$
a \begin{array}{llll}
a & a(b a)^{n / k-1} & b & a(b a)^{k-1} .
\end{array}
$$

Then, $\theta$ gives rise to a configuration of two symmetries whose product has order $n$ and has $2 u+1$ or $2 u+3$ fixed points on a Riemann surface of genus $g=(u+2) n-u-$ $n / \mu-n / k-(k-1) / 2$. 
ACKNOWLEDGMENTS. The authors are grateful to a referee for his helpful and accurate comments.

\section{REFERENCES}

1. E. Bujalance, A. F. Costa and D. Singerman, Application of Hoare's theorem to symmetries of Riemann surfaces, Ann. Acad. Sci. Fenn. 18(1993), 307-322.

2. M. Izquierdo and D. Singerman, Pairs of symmetries of Riemann surfaces, Ann. Acad. Sci. Fenn. 23(1998), 3-24.

3. M. Izquierdo and D. Singerman, On the fixed point set of automorphisms of nonorientable surfaces without boundary, Geometry Topology Monographs, 1 (the Epstein birthday schrift) (1998) 295-301.

4. A. M. Macbeath, Action of automorphisms of a compact Riemann surface on the first homology group, Bull. London Math. Soc. 5(1973), 103-108.

5. S. M. Natanzon, Finite groups of homeomorphisms of surfaces and real forms of complex algebraic curves, Trans. Moscow Math. Soc. 51(1989), 1-51.

6. G. Gromadzki, On a Harnack-Natanzon theorem for the family of real forms of Riemann surfaces, J. Pure Appl. Algebra 121(1997), 253-269.

7. D. Singerman, On the structure of non-Euclidean crystallographic groups, Proc. Camb. Phil. Soc. 76(1974), 233-240. 